\title{
The Evolution of the Immune System
}

Conservation and Diversification

\section{Davide Malagoli}

Department of Life Sciences

Biology Building, University of Modena

and Reggio Emilia, Modena, Italy 